\title{
Non-Markovian Character in Human Mobility: Online and Offline
}

\author{
Zhi-Dan Zhao ${ }^{1}$, Shi-Min Cai ${ }^{1,2, *}$, Yang Lu ${ }^{1}$ \\ 1 Web Sciences Center, School of Computer Science and Engineering, University of \\ Electronic Science and Technology of China, Chengdu 611731, P. R. China \\ 2 State Key Laboratory of Networking and Switching Technology, Beijing University of \\ Posts and Telecommunications, Beijing, 100876, P. R. China \\ * E-mail: shimin.cai81@gmail.com
}

\begin{abstract}
The dynamics of human mobility characterizes the trajectories humans follow during their daily activities and is the foundation of processes from epidemic spreading to traffic prediction and information recommendation. In this paper, we investigate a massive data set of human activity including both online behavior of browsing websites and offline one of visiting towers based mobile terminations. The non-Markovian character observed from both online and offline cases is suggested by the scaling law in the distribution of dwelling time at individual and collective levels, respectively. Furthermore, we argue that the lower entropy and higher predictability in human mobility for both online and offline cases may origin from this non-Markovian character. However, the distributions of individual entropy and predictability show the different degrees of non-Markovian character from online to offline cases. To accounting for non-Markovian character in human mobility, we introduce a protype model with three basic ingredients, preferential return, inertial effect, and exploration to reproduce the dynamic process of online and offline human mobility. In comparison with standard and biased random walk models with assumption of Markov process, the proposed model is able to obtain characters much closer to these empirical observations.
\end{abstract}

\section{Introduction}

Uncovering the dynamics of human mobility is pivotal to decipher the behavior patterns of human daily activities, with widely practical applications ranging from epidemic containment [1 5] to traffic predication 6, 7] and information recommendation 8, 9. The pursuit has been facilitated greatly by advances in information technology, especially by the availability of massive Internet data and resources including the real-time tracking trajectories of human mobility at temporal and spatial scales [10. Large amounts of studies on human mobility are therefore generated based on these trajectories in physical space, such as dollar dispersal [1], GPS 12, mobile phone 7, 13 15, questionnaire [16, or cyber space, such as online web surfing [17,18 and interest transition 19, 20]. In these works, several widely accepted indicators, e.g., the trip distance distribution, the radius of gyration, the number of visited locations over time, the human mobility motifs, the consecutively dwelling time (or click length) for a location, the entropy and predictability, are used to statistically characterize the dynamics of human mobility [11, 13 15, 18, $19,21$.

Since Einstein's study 22 on the motions of small particles suspended in liquids, diffusion and random walk have been seemed as a paradigm describing and modeling the mobility in many cases of physical and biological domains ranging from charge transport in disordered conductors to foraging patterns of animals and fishes 23, 24. On the other hand, a widely used assumption of Markovian (memoryless) dynamics is deemed to be existence in online human activities, which suggests that click actions are independent each other 25,27. However, recent evidences $19,28,29$ demonstrate that online human activities may obey a non-Markov process.

Although many studies have focused on human mobility recently, it is still obscure that whether 
individual mobilities for both online and offline cases exactly follow a Markov process and why there are lower entropy and higher predictability in human mobility. In this paper, our systematical analysis of a massive data set commonly including both online and offline human activities for same individuals seeks to more clearly unveil the dynamics of human mobility. The dynamic processes of human mobility for both online and offline cases are translated into visual networks describing that the participants transit from one website (or tower) to another. Based on that, both at individual and collective levels, the heterogeneous rank distributions of nodes (i.e., websites and towers) according to visit frequency suggest that the human mobility follows a nonuniform process and the scaling law in the distribution of dwelling time shows the non-Markovian character in human mobility. Furthermore, the non-Markovian character imply the existence of memory in dynamics of human mobility, leading to a lower entropy and higher predictability in human mobility. In turn, the diverse distributions of individual entropy and predictability indicate the different degree of non-Markovian character between online and offline cases. By comparing with two Markov processes, Standard Random Walk (SRW) and Biased Random Walk (BRW), we account for the non-Mrakovian character and origin of lower entropy and higher predictability in human mobility based on a protype model with three basic ingredients, preferential return, inertia effect and exploration, underlying mechanisms governing the dynamics of online and offline human mobility. Our results to some extent uncover that the underlying mechanism governing the dynamics of human mobility is generic no matter of the temporal and spacial differences among their activities.

\section{Results}

\section{Empirical observations}

We explore a massive data set of human activity including online behavior of browsing websites and offline one of visiting towers based mobile terminations, whose trajectories were recorded by sequences of websites and towers at temporal scale, respectively. The non-Markovian character in human mobility is investigated with these aspects, 1) visual networks of human mobility; 2) rank distributions of nodes and distributions of dwelling time at individual and collective levels, respectively; 3 ) the individual entropy and predictability in human mobility over whole duration.

Visual network of human mobility. An individual can visit different websites (towers) during her/his communicating activity based mobile termination. A convenient way to represent the direct associations among them is to construct a visual network where nodes denote websites (towers) and links correspond to observed transitions among websites (towers), shown in Fig. 11(a) and (b) respectively. The node sizes and link weights are determined by visit and transition frequency respectively, and the self-loops of nodes indicates the dwelling time. From Fig. 1(a) and (b), we can find that the networks of human mobility are obviously heterogeneous, suggesting the human mobility is a nonuniform process.

Rank distribution of nodes. At individual level, the nodes are visited in different frequency, leading to heterogeneous node sizes. We rank nodes according to their size and probe its rank distribution. In Fig 2(a) and (b), the rank distributions for both online and offline cases are approximately fitted by an exponentially truncated power-law formula, $f_{r}=r^{-\beta} \exp (-r / \kappa)$, with values of $\beta$ are 0.5 and 1.15 for online and offline cases, respectively (the values of the exponent $\beta$ are tested by Kolmogorov-Smirnov (KS) test). Note that this truncated fat-tailed distribution is robust in respect to other individuals (see Fig. S1 in Supplementary Information). For whole system, we are also interesting that the rank distribution of nodes when their sizes are aggregated at collective level. As the intensity of individual activity is different, we scaled the aggregated node size with the number of participants who visit it to filter its effect. In Fig 2(c) and (d), the rank distributions for both online and offline cases at collective level shows a power-law formula, $f_{r}=r^{-\beta}$, similar to the Zip'f law characterizing the rank distribution of locations in human mobility $13,14,30$. The values of $\beta, 1.57$ and 1.31 obtained from maximum likelihood estimation, are close with each other. Additionally, compared with that at individual level, the scaling 
law at collective level reduce the exponential truncation, which may result from the remove of effect of intensity of individual activity. These heterogeneous rank distributions of nodes at two level suggest the nonuniformity of dynamic process of human mobility and may imply the non-Markovian charter in human mobility. Simultaneously, considering the similar pattern of human mobility for online and offline cases at two levels, we state that their underlying mechanisms governing the dynamics of human mobility may be same.

Distribution of dwelling time. Many approaches have been proposed to quantify human mobility, yet here we are interesting in the dwelling time $l$ consecutively visiting same node as a result of taking advantage of the nature of enough large data set. Additionally, the dwelling time associates with the inter-arrival time, whose statistical distribution can characterizes the Markovian property of stochastic process [31. Like the analysis of rank distribution of node, we first focus on the statistical measurements of dwelling time for both online and offline cases at individual level. A group of typical sequences of $l$ corresponding to online and offline cases are shown Fig. 3(a) and (b), respectively. They both oppose some extremely large values and behave a clustering behavior, which result in their power-law distributions presented in Fig. 3(c) and (d). Much more concretely, for the distribution $P(l) \sim l^{-\alpha}$, a larger $\alpha$ indicates a much more irregular sequence of $l$, and the fat tail suggests that participant tends to take abnormally long time consecutively visiting same node. By fitting the power-law distributions based on maximum likelihood estimation [46, we obtain $\alpha \approx 1.96$ and 1.36 for online and offline cases, respectively, confirmed by the irregularity of sequence shown in Fig. 3(a) and (b). Moreover, the scaling law proves the existence of non-Markovian character in human mobility because Markov process shows exponential 31]. Note that much more participants from this massive data set have been measured to confirm the stability of scaling law (see Fig. S2 in Supplementary Information).

On the other hand, we also aggregate $l$ from all participants to quantify the non-Markovian character at collective level. As shown in Figs. 4 (a) and (b), it can be found that the distributions of $l$ for online and offline cases follow power-law form at multiply scales although a little deviation exists at top scale. It is easy to understand that participant has inertia to stay at a particular node, resulting in the powerlaw distributions of dwelling time. Additionally, at collective level, the values of $\alpha$ obtained through fitting the the power-law distributions for both online and offline cases at multiply scales are almost same, demonstrating the non-Markovian character generally roots in human mobility and suggesting the generic mechanism governing dynamics of human mobility no matter of its different behavior.

Entropy and predictability. Entropy is the most fundamental quantity describing the uniformity of system and the degree of predictability from time series 15 . A lower value of entropy implies the intense non-uniformity of system and higher predictability, and vice versa. Here, we discuss how to measure the entropy of sequence (i.e.,trajectories) in human mobility. For this purpose, an estimator based on Lempel-Ziv data compression algorithm [15, 32,33] is used to obtain the entropy of a given sequence with $N_{a}$ actions as follows,

$$
E \approx\left(\frac{1}{N_{a}} \sum_{j} \Lambda_{j}\right)^{-1} \ln N_{a} .
$$

where $\Lambda_{j}$ is the length of the shortest substring starting at position $j$ which doesn't previously appear from position 1 to $j-1$. Kontoyianis et al. 32 33 have proved that $E$ converges to the actual entropy as $N_{a}$ approaches infinity. We compute the values of $E$ over all participants for both online and offline cases. Figure 5(a) and (b) present their distributions of entropy respectively, whose averages are different from $\langle E\rangle \simeq 0.69$ for online case to $\langle E\rangle \simeq 0.35$ for offline case. However, the low entropy are both observed in these two cases, suggesting higher predictability in human mobility.

Subjecting to Fano's inequality in [34], the predictability $\Pi^{\max }(E, S)$ when individual with entropy $E$ moves between $S$ nodes is determined by

$$
E=-\left[\Pi^{\max } \log _{2} \Pi^{\max }+\left(1-\Pi^{\max }\right) \log _{2}\left(1-\Pi^{\max }\right)\right]+\left(1-\Pi^{\max }\right) \log _{2}(S-1) .
$$

We compute $\Pi^{\max }$ separately for each participant for online and offline cases, and show their distributions 
shown in Figs. 5(c) and (d). It can be found that the higher predictability is presented for online and offline cases, and $\langle\Pi\rangle$ is 0.94 and 0.97 respectively well agreeing with the lower entropy. Combined with non-Markovian character in human mobility, we state that the high predictability may be as a result of memory and non-randomness in dynamics of human mobility.

\section{Model and Simulation}

The observed non-Markovian character as well as low entropy and high predictability infer that a participant visits to next node is unlikely to be random and governed by underlying mechanism. Thus, it is meaningful to learn that why these scaling laws emergence from human mobility and what is the underlying mechanism governing the dynamics of human mobility with the low entropy and high predictability. Then, to acquire a reasonable mechanism characterizing non-Markovian dynamics, three ingredients derived from empirical results, i.e., preferential return, exploration and inertia, are employed to depict a protype model. A schematic illustration of proposed model is shown in Fig. 6(a). Concretely speaking, a participant has two alternative choices, exploring a new node and revisit one of previous nodes. The probability, making a choice of exploration, $\rho n^{-\lambda}$, is determined by statistical analysis of all participants, whereas the complementary probability $1-\rho n^{-\lambda}$ is for another choice. Herein, the values of $\rho$ and $\lambda$, estimated from real data sets, are 0.49 and 0.51 for online case and 0.39 and 0.22 for offline case (see

Fig. S3 in Supplementary Information). When revisiting previous nodes, each of them has a probability of preferential return. Therefore, an assumption, known in network science and human mobility $14,20,35$, is that participants revisit one particular node according to the prior visiting probability:

$$
\Pi=\frac{f_{i}}{\sum_{j=1}^{S} f_{j}},
$$

where $f_{i}$ is the frequency of visiting node $i$, i.e., the accumulated dwelling time at node $i$. Once a particular node is chosen, she/he can keep staying at the selected node for a time interval as a result of her/his inertia effect characterized by dwelling time. And the time interval can be simulated by an excited random walk (ERW) with tunable parameter $p$ [36], that is, it equals to the time that the walker first returns to the starting point. As the walker eventually return to the starting point with probability 1 and his first return time $t$ decays as $t^{-(2-p)}$, ERW associates with the non-Markovian character of the dwelling time. Note that $p$ is nonnegative and usually less than 1 .

For the comparison between the proposed model and those models with hypothesis of Markovian dynamics, we introduce two models, standard random walk (SRW) visiting next node with uniform probability and biased random walk (BRW) visiting next node subjecting to unequal chances. Based on all three models, the simulations are independently performed with 20,000 agents, each evolves $10^{6}$ time steps. The numerical results are shown in Fig. 6(b)-(d). On one hand, the scaling law predicted the proposed model with different $p$ are much closer to empirical result comparing with SRW and BRW, although its values are a little departure from these empirical ones. (see in Fig. 6(b)). On the other hand, the lower entropy and higher predictability obtained from the proposed model well belong to the ranges observed from all participants, while those of SRW and BRW totally deviate from these ranges. These results suggesting these two Markovian models are not able to perfectly describe human mobility for both online and offline cases.

\section{Discussion}

Despite making large advances in human dynamics, especially human mobility $7,13,16,37,42]$, we still pay much attention on its dynamic characters because of the obscurity of generical mechanism. Through 
analyzing a massive data set including both online and offline trajectories of human mobility, the nonuniform dynamic process of human mobility is shown by the heterogenous rank distribution of nodes of visual network, and its non-Markovian character is suggested by the emergence of scaling laws in distributions of dwelling time at both individual and collective levels. The non-Markovian character implies the memory in dynamics of human mobility, leading to the low entropy and high predictability. Furthermore, a protype model involved with three ingredients, preferential return, exploration and inertia is introduced to reproduce transition process of human mobility and account for the origin of empirical results. The simulations show that the proposed model presents a better prediction of scaling law comparing with those models with Markovian dynamics, and behaves the lower entropy and higher predictability close to statistical average for all participants. Note that the present model may be so simple as to be not able to unveil extra dynamic characters but enough to figure out the non-Markovian characters.

The scaling laws uncovered from data and the protype model developed accordingly can be applied to addressing significant problems ranging from human-behavior prediction and the design of search algorithms [3, 14] to controlling spreading processes [8, 43. Moreover, the non-Markovian character of human mobility could make to deeply understand other studies such as synchronization [44] and temporal networks 45. As a demonstration, we have probed the degree of predictability of human behavior and its origin.

\section{Materials and Methods}

\section{Data sets description.}

The massive data set used is from a large-scale real communication system based mobile termination, where participant browses websites when they are moving from one place to another. Thus, it includes two groups of records, online trajectory of browsing weibsites and offline trajectory of visiting towers. In order to improve the quality of trajectory reconstruction, we remain these participants whose trajectory achieves at least 20 distinct websites and towers, and the average visiting times to each website or tower is more than 10. Finally, the total number of participants is 8,929 .

\section{Maximum likelihood estimation and Kolmogorov-Smirnov test.}

Maximum Likelihood Estimation is a solid tool for learning parameters as a data mining model. Its principle makes the desired probability distribution be the best fit of observed data, and its methodology tries to do two things. First, it is a reasonably well-principled way to work out what computation you should be doing when you want to learn some kinds of model from data. Second, it is often fairly computationally tractable. The details of the maximum likelihood method have been widely published. Herein, we perform such fits according to [46].

In statistics, Kolmogorov-Smirnov (KS ) test is a nonparametric methodology that compares either an observed distribution, $S(x)$, with a theoretical distribution, $F^{*}(x)$, or two observed distributions. In either case, the procedure is involved with forming the cumulative distributions of $S(x)$ and $F^{*}(x)$ and finding the size of the largest difference between them. The KS test is based on the following test statistic:

$$
D=\sup \left|F_{c}^{*}(x)-S_{c}(x)\right|
$$

where smaller $D$ values correspond to better fit with theoretical distribution.

\section{Standard, biased and excited random walks}

For standard Random Walk (SRW), $S$ distinct nodes are distributed and then the walker select one node to visit randomly (with probability $1 / S$ ) at each step. The process is repeated until all of the $S$ 
nodes are visited. While for biased random walk (BRW), the walker select one node to visit at each step according to the preferential probability $\Pi_{i}=f_{i} / \sum f_{j}$ depending on its visit frequency [14 35. The large difference between SRW and BRW is that SRW can bring larger entropy and lower predictability than BRW. However, both of them cannot simultaneously include the low entropy and high predictability observed from empirical data.

The excited random walk (ERW) on one dimension represents a simple and surprisingly rich example of a non-Markov process and possesses a basic feature that transitory nature of the bias [36]. For any $p<1$, it is recurrent, that is, the walker revisits sites unlimited and the probability to eventually return to the starting point equals to 1 . Its process on the infinite chain of sites is simply described as: the walker, starting from a certain site to first visit, hops to the right with probability $p$ and to the left with complementary probability $1-p$; then if the walker hops to a previously visited site, at next time step the hopping probabilities to the left and right are both $1 / 2$, otherwise they are biased; the walker finally stops hopping until he first return to the starting site. The time steps across a whole ERW are deemed as the time interval of inertial effect.

\section{Acknowledgments}

This work is jointly supported by the NNSFC (Grant Nos.91024026 and 61004102), Special Project of Sichuan Youth Science and Technology Innovation Research Team (No.2013TD0006), and the Fundamental Research Funds for the Central Universities (Grant Nos.ZYGX2011YB024 and ZYGX2012J075). SMC acknowledgments the financial support of the Open Foundation of State key Laboratory of Networking and Switching Technology (Beijing University of Posts and Telecommunications) (Grant No.SKLNST2013-1-18) and the startup fund of UESTC. 


\section{References}

1. Hufnagel L, Brockman D, Geisel T (2004) Forecast and control of epidemics in a globalized worlds. Proc Natl Acad Sci USA 101: 15124.

2. Colozza V, Barrat A, Barthelemy M, Vespignani A (2007) Predictability and epidemic pathways in global outbreaks of infrectous diseases: the SARS case study. BMC Med 5: 34.

3. Vespignani A (2009) Predicting the behavior of techno-social systems. Science 325: 425.

4. Wang P, Gonzalez MC, Hidalgo CA, Barabási AL (2009) Undersatanding the spreading patterns of mobile phone viruses. Science 324: 1071

5. Yang H, Tang M, Zhang H F (2013) Efficient community-based control strategies in adaptive networks. New J Phys 14: 123017.

6. Helbing D (2001) Traffic and related self-driven many-particle systems. Rev Mod Phys 73: 10671141.

7. Simini F, González MC, Maritan A, Barabási AL (2012) A universal model for mobility and migration patterns. Nature 484: 96-100.

8. Balcan D, Vespignani A (2011) Phase transitions in contagion processes mediated by recurrent mobility patterns. Nat Phys 7: 581-586.

9. Lü L, Medo M, Yeung CH, Zhang YC, Zhang ZK, et al. (2012) Recommender systems. Phys Rep 519: $1-49$.

10. Starnini M, Baronchelli A, Pastor-Satorras R (2013) Modeling human dynamics of face-to-face interaction networks. Phys Rev Lett 110: 168701.

11. Brockmann D, Hufnagel L, Geisel T (2006) The scaling laws of human travel. Nature 439: 462-465.

12. Rhee I, Shin M, Hong S, Lee K, Chong S (2012) On the levy-walk nature of human mobility. In: INFOCOM'08. pp. 924-932.

13. Gonzalez MC, Hidalgo CA, Barabási AL (2008) Understanding individual human mobility patterns. Nature 453: 779-782.

14. Song C, Koren T, Wang P, Barabási AL (2010) Modelling the scaling properties of human mobility. Nat Phys 6: 818-823.

15. Song C, Qu Z, Blumm N, Barabási AL (2010) Limits of predictability in human mobility. Science 327: $1018-1021$.

16. Yan X Y, Han X P, Wang B H, Zhou T (2013) Diversity of individual mobility patterns and emergence of aggregated scaling laws. Sci. Rep 3: 2678.

17. Huberman BA, Pirolli PLT, Pitkow JE, Lukose RM (1998) Strong regularities in world wide web surfing. Science 280: 95-97.

18. Chmiel A, Kowalska K, Hołyst J (2009) Scaling of human behavior during portal browsing. Phys Rev E 80: 066122.

19. Zhao ZD, Yang Z, Zhang Z, Zhou T, Huang ZG, Lai YC. (2013) Emergence of scaling in humaninterest dynamics. Sci. Rep. 3: 3472. 
20. Zhao ZD, Gao YC, Cai SM (2013) The dynamic pattern of human attention. arXiv preprint arXiv:13084847 .

21. Schneider CM, Belik V, Couronn T, Smoreda Z, Gonzlez MC (2013) Unravelling daily human mobility motifs. J R Soc. Int. 10.

22. Einstein A (1905) On the movement of small particles suspended in stationary liquids required by the molecular-kinetic theory of heat. Annalen der Physik 17: 16.

23. Bouchaud JP, Georges A (1990) Anomalous diffusion in disordered media: statistical mechanisms, models and physical applications. Phys Rep 195: 127-293.

24. Ben-Avraham D, Havlin S (2000) Diffusion and reactions in fractals and disordered systems. Cambridge University Press.

25. Brin S, Page L (1998) The anatomy of a large-scale hypertextual web search engine. Comp Net ISDN 30: 107-117.

26. Craswell N, Szummer M (2007) Random walks on the click graph. In: Proc. 30th Annual Int. ACM SIGIR CRDIR. ACM, pp. 239-246.

27. Fagin R, Karlin AR, Kleinberg J, Raghavan P, Rajagopalan S, et al. (2001) Random walks with" back buttons". Ann Appl Probab : 810-862.

28. Meiss MR, Gonçalves B, Ramasco JJ, Flammini A, Menczer F (2010) Agents, bookmarks and clicks: a topical model of web navigation. In: Proc. 21st ACM CHH. ACM, pp. 229-234.

29. Chierichetti F, Kumar R, Raghavan P, Sarlós T (2012) Are web users really markovian? In: Proc. 21st Int. Conf. WWW. ACM, pp. 609-618.

30. Bagrow JP, Lin YR (2012) Mesoscopic structure and social aspects of human mobility. PLoS ONE 7: e37676.

31. Kingman JFC (1963) The exponential decay of markov transition probabilities. Proc London Math Soc 3: $337-358$.

32. Ziv J, Lempel A (1977) A universal algorithm for sequential data compression. IEEE T Inform Theory 23: 337-343.

33. Kontoyiannis I, Algoet PH, Suhov YM, Wyner AJ (1998) Nonparametric entropy estimation for stationary processes and random fields, with applications to english text. IEEE T Inform Theory 44: $1319-1327$.

34. Fano R, Hawkins D (1961) Transmission of information: A statistical theory of communications. Am J Phys 29: 793.

35. Barabási AL, Albert R (1999) Emergence of scaling in random networks. Science 286: 509-512.

36. Antal T, Redner S (2005) The excited random walk in one dimension. J Phys A 38: 2555.

37. Barabási AL (2005) The origin of bursts and heavy tails in human dynamics. Nature 435: 207-211.

38. Oliveira JG, Barabási AL (2005) Human dynamics: Darwin and einstein correspondence patterns. Nature 437: 1251-1251.

39. Dezsö Z, Almaas E, Lukács A, Rácz B, Szakadát I, et al. (2006) Dynamics of information access on the web. Phys Rev E 73: 066132. 
40. Zhou T, Kiet HAT, Kim BJ, Wang BH, Holme P (2008) Role of activity in human dynamics. Europhys Lett 82: 28002.

41. Gonçalves B, Ramasco JJ (2008) Human dynamics revealed through web analytics. Phys Rev E 78.

42. Wu Y, Zhou C, Xiao J, Kurths J, Schellnhuber HJ (2010) Evidence for a bimodal distribution in human communication. Proc Natl Acad Sci U S A 107: 18803-18808.

43. Zhao ZD, Liu Y, Tang M (2012) Epidemic variability in hierarchical geographical networks with human activity patterns. Chaos 22: 023150.

44. Arenas A, Diaz-Guilera A, Kurths J, Moreno Y, Zhou C (2008) Synchronization in complex networks. Phys Rep 469: 93-153.

45. Holme P, Saramäki J (2012) Temporal networks. Phys Rep 519: 97-125.

46. Clauset A, Shalizi C, Newman MEJ (2009) Power-law distributions in empirical data. SIAM Rev 51: $661-703$. 
Figure Legends 

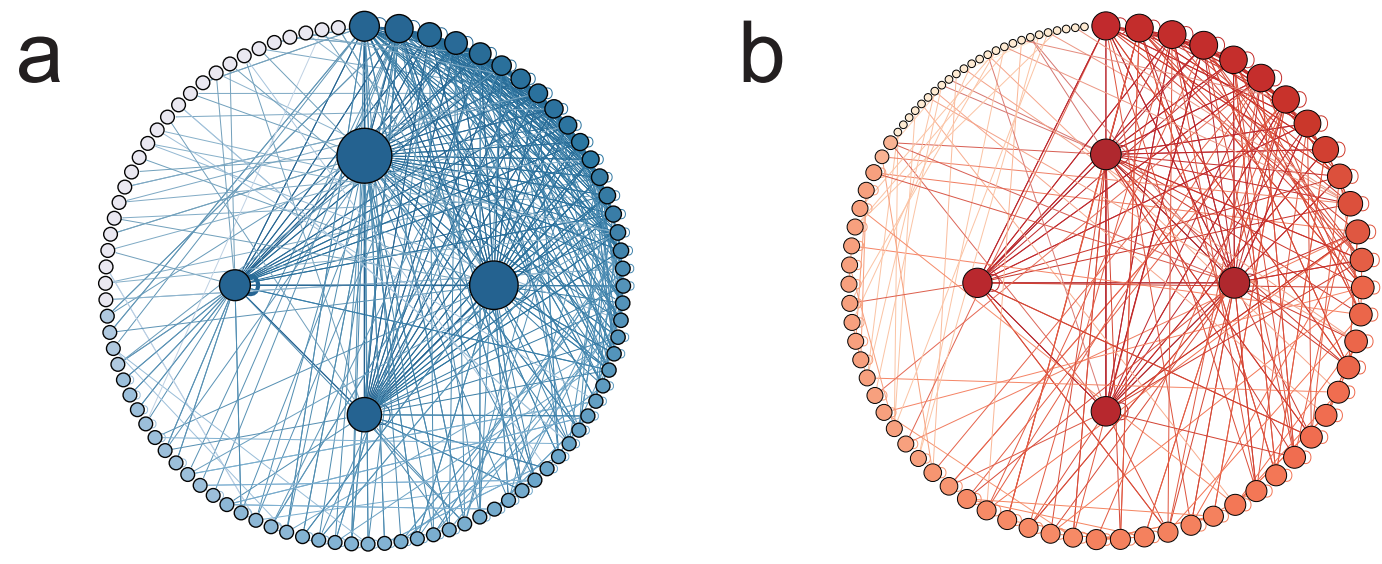

Figure 1. Visual network of human mobility. For a selected participant, the visual networks for online (a) and offline (b) cases describe the dynamic processes of browsing distinct websites and visiting different towers, respectively. In these networks, node denotes website or tower and weighted link indicates the intensity of transition. Node size is determined by its visit frequency and self-loop represents the repeat visits to the same node. A few frequently visited node are marked in the central area of network. The numbers of clicks $\left(N_{a}\right)$ for two cases are 6,666 and the number of nodes are 95 . 

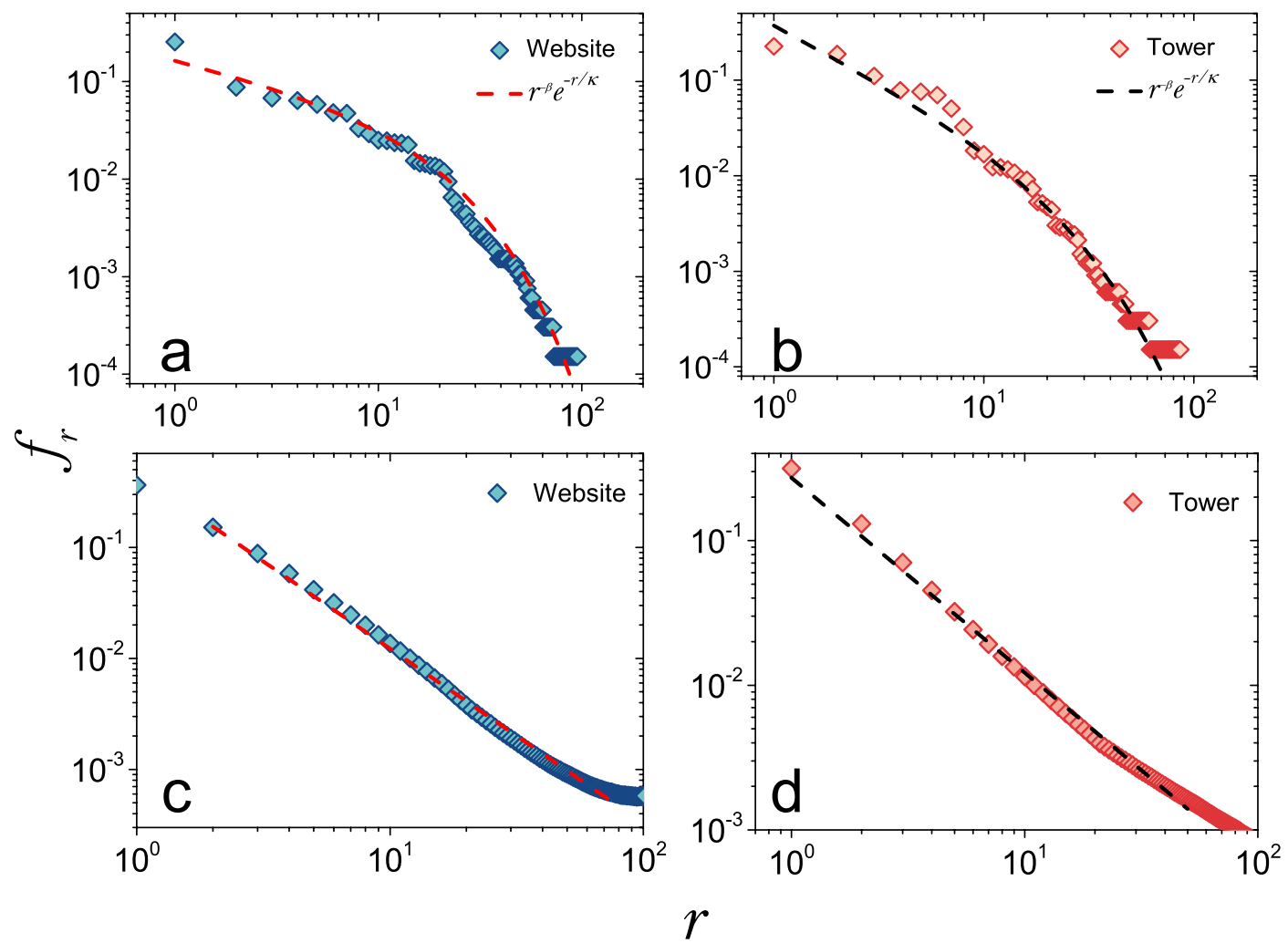

Figure 2. Rank distribution of nodes of visual networks. The rank distribution at individual level shows the truncated power-law scaling, $f_{r} \propto r^{-\beta} \exp (-r / \kappa)$, where the fitted values of the exponent $\beta$ and $\kappa$ are $(\beta, \kappa)=(0.50,20)$ for online case (a) and $(\beta, \kappa)=(1.15,20)$ for offline case (b). For the whole system, we scaled the aggregated node size with the number of participants who visit it and rank them. The rank distribution at collective level becomes a power-law scaling, $\beta=1.57 \pm 0.02$ for online case (c) and $\beta=1.31 \pm 0.01$ for offline case (d). The similar pattern of human mobility for online and offline cases at two levels suggests that their underling mechanisms governing the dynamics 

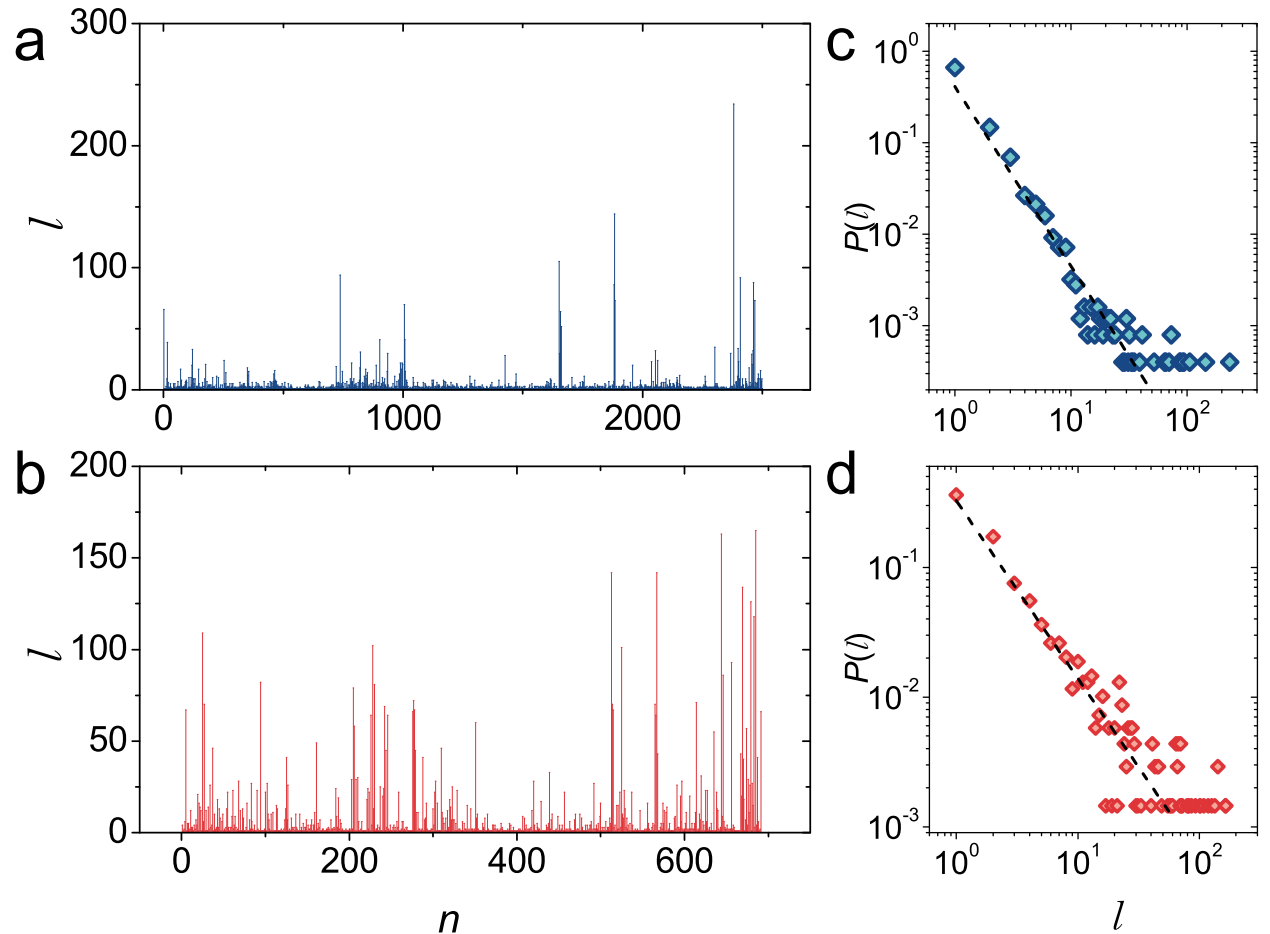

Figure 3. The sequence of dwelling time and its probability distribution at individual level. The sequences $l$ of dwelling time in respect to click $n$ for online (a) and offline (b) cases, obtained from the same participant, show the burst and clustering behavior. The corresponding probability distribution $P(l)$ follows power-law scaling, $P(l) \sim l^{-\alpha}$, where the exponent $\alpha$, with exponents $\alpha \approx 1.96$ and 1.36 for online (c) and offline (d) cases, respectively. The values of the exponent $\alpha$ are estimated by using the maximum likelihood criterion [46]. These empirical results suggest the non-Markovian character in human mobility. 

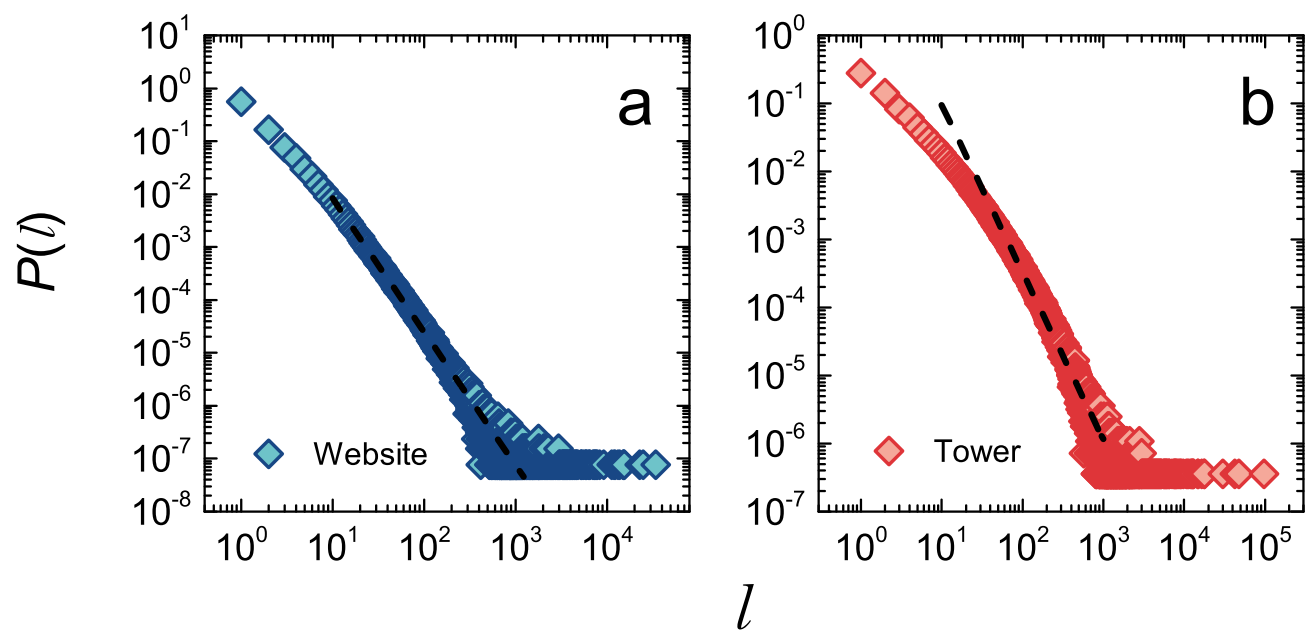

Figure 4. The probability distribution of dwelling time at collective level. By aggregating the dwelling time $l$ from all participants of whole system, we show their probability distribution at collective level for online (a) and offline (b) cases, respectively. Both of them can be fitted by $P(l) \sim l^{-\alpha}$ with the close exponent, i.e., $\alpha=2.51 \pm 0.02$ for online case and $\alpha=2.46 \pm 0.02$ for offline case. 

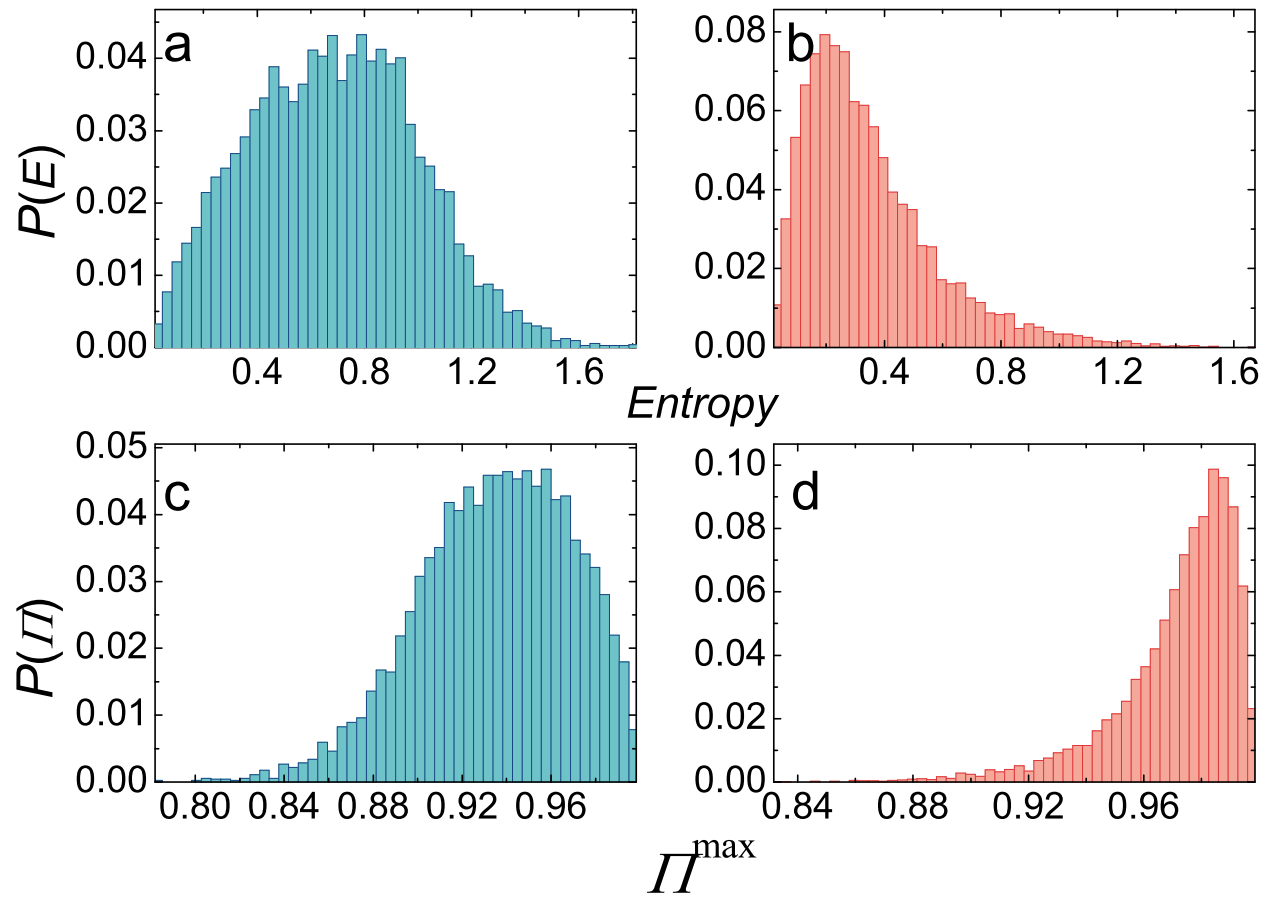

Figure 5. The probability distributions of individual entropy and predictability. For all participants of whole system, the probability distributions of entropy with the average $\langle E\rangle \simeq 0.69$ and 0.35 for online (a) and offline (b) cases are presented to characterize the intense nonuniformity of system. In turn, the probability distributions of corresponding predictability with the average $\langle\Pi\rangle \simeq 0.94$ and 0.97 for online (c) and offline (d) cases confirm the lower entropy. Combined with the non-Markovian character in human mobility, the higher predictability may be as a result of memory and non-randomness in dynamics of human mobility. 


\section{a}

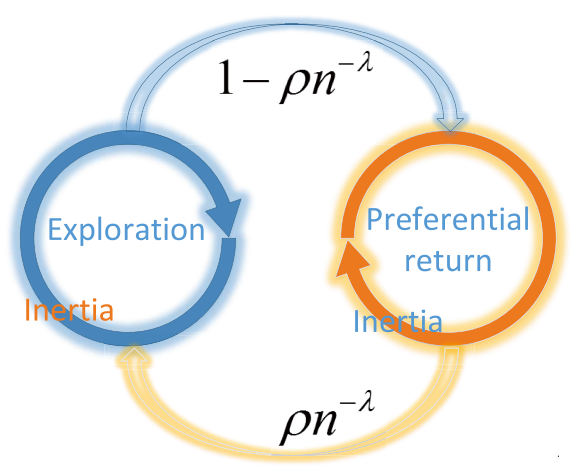

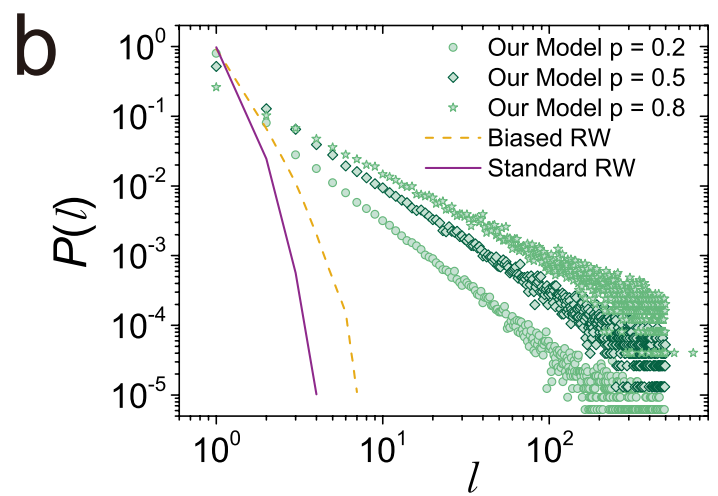
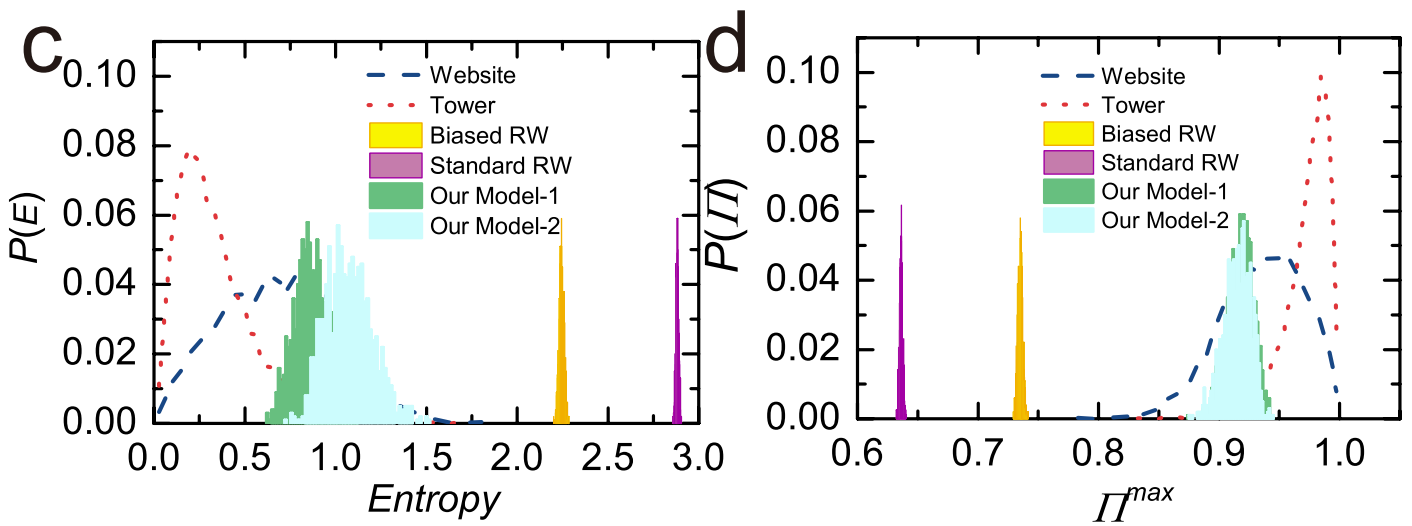

Figure 6. The schematic illustration of the proposed model of human mobility and the simulations. (a) Schematic illustration of the model, where an individual can enter one of the two dynamically complementary states at each hopping step: exploring a new node with the probability $\rho n^{-\lambda}$ (the state of "Exploration") or returning preferentially to a previously visited node with the probability $1-\rho n^{-\lambda}$ (the state of "Preferential return"). Regardless of which state occurring, a selected node is plugged by the inertial effect, a microscopic process modeled by ERW with tunable parameter $p$. These experimental results are obtained from 20,000 independent model simulations, each using $10^{6}$ 